\title{
Poly(ADP-Ribose) Polymerase Activation Induces High Mobility Group Box 1 Release From Proximal Tubular Cells During Cisplatin Nephrotoxicity
}

\author{
Jinu KIM ${ }^{1,2}$ \\ ${ }^{1}$ Department of Anatomy, Jeju National University School of Medicine, Jeju, Republic of Korea, \\ ${ }^{2}$ Department of Biomedicine and Drug Development, Jeju National University, Jeju, Republic \\ of Korea
}

Received October 23, 2014

Accepted April 29, 2015

On-line October 8, 2015

\begin{abstract}
Summary
Cisplatin is one of the most potent chemotherapy drugs against cancer, but its major side effect such as nephrotoxicity limits its use. Inhibition of poly(ADP-ribose) polymerase (PARP) protects against various renal diseases via gene transactivation and/or ADP-ribosylation. However, the role of PARP in necrotic cell death during cisplatin nephrotoxicity remains an open question. Here we demonstrated that pharmacological inhibition of PARP by postconditioning dose-dependently prevented tubular injury and renal dysfunction following cisplatin administration in mice. PARP inhibition by postconditioning also attenuated ATP depletion during cisplatin nephrotoxicity. Systemic release of high mobility group box 1 (HMGB1) protein in plasma induced by cisplatin administration was significantly diminished by PARP inhibition by postconditioning. In in vitro kidney proximal tubular cell lines, PARP inhibition by postconditioning also diminished HMGB1 release from cells. These data demonstrate that cisplatin-induced PARP1 activation contributes to HMGB1 release from kidney proximal tubular cells, resulting in the promotion of inflammation during cisplatin nephrotoxicity.
\end{abstract}

\section{Key words}

Cisplatin nephrotoxicity • Poly(ADP-ribose) polymerase $\bullet$ Necrosis

- High mobility group box $1 \bullet$ Kidney

\section{Corresponding author}

J. Kim, Department of Anatomy, Jeju National University School of Medicine, 102 Jejudaehak-ro, Jeju, Republic of Korea 690-756. Fax: +82-64-702-2687. E-mail: jinu.kim@jejunu.ac.kr

\section{Introduction}

Cisplatin is the most commonly used drug for treatment of malignant tumors in testis, ovary, breast, and many other tissues (Siddik 2003, Wang and Lippard 2005). However, it has severe side effects in normal tissue, such as ototoxicity, neurotoxicity, nausea, and nephrotoxicity (Stark and Howel 1978). Among them, cisplatin nephrotoxicity is a major side effect, which occurs in approximately one-third of the patients treated with cisplatin. Clinically, cisplatin nephrotoxicity is characterized by renal dysfunction, represented by lower glomerular filtration rate and higher serum creatinine (Arany and Safirstein 2003, Beyer et al. 1997). Although pathophysiological research on cisplatin nephrotoxicity has been carried out for the last three decades, the cellular and molecular mechanisms remain unclear. Especially, apoptosis of renal tubular cells has so far been a major focus of mechanistic investigation of cisplatin nephrotoxicity, whereas tubular cells undergoing necrosis during cisplatin nephrotoxicity have been poorly investigated.

Poly(ADP-ribose) polymerase (PARP) as a nuclear enzyme plays an important role in regulating protein functions through poly(ADP-ribosyl)ation and gene transactivation. PARP catalyzes the transfer of ADP-ribose from nicotinamide adenine dinucleotide and conjugates poly(ADP-ribose) onto various proteins as well as PARP itself, thus leading to a variety of physiological processes including modulation of protein 
functions and protein-protein interactions (Kraus and Lis 2003, Krishnakumar et al. 2008). Alternatively, excessive PARP activation leads to necrotic cell death via depletion of intracellular adenosine triphosphate (ATP) (Ha and Snyder 1999, Moubarak et al. 2007). It has been reported that pharmacological and genetic inhibition of PARP protects kidneys against ischemia-reperfusion injury (Devalaraja-Narashimha and Padanilam 2009, Martin et al. 2000), diabetes (Shevalye et al. 2010), and ureteral obstruction (Kim and Padanilam 2011). In a cisplatin nephrotoxicity model, it was found that PARP gene deletion and preconditioning treatment with a PARP inhibitor are renoprotective via nuclear factor- $\kappa \mathrm{B}$, c-Jun $\mathrm{N}$-terminal kinase and p38 mitogen-activated protein kinase activation (Kim et al. 2012). The previous reports mainly examined PARP-mediated gene transactivation in various renal diseases, whereas the contribution of PARP-mediated necrosis to inflammation, especially in cisplatin nephrotoxicity, remains incompletely understood.

Necrotic cells have been reported to release high mobility group box 1 (HMGB1) protein into the extracellular space. Since HMGB1-deficient cells are unable to activate macrophages, HMGB1 is thought to be a key mediator of inflammation (Scaffidi et al. 2002). Originally, HMGB1 is an architectural chromatin-related protein and one of the most abundant proteins in the nucleus (Thomas and Travers 2001). However, extracellular HMGB1 can mediate inflammation through direct binding to a variety of receptors on innate immune cells (Lotze and Tracey 2005). Although HMGB1 release from necrotic cells is occurred by passive diffusion, the mechanism of HMGB1 release during necrosis is undefined. This study investigated the contribution of PARP activation to HMGB1 release from renal tubular cells undergoing necrosis during cisplatin nephrotoxicity. To extend this finding in a clinical context, we treated a PARP inhibitor PJ34 based on a modified phenanthridinone structure which is approximately 10,000 times more potent than the prototypical PARP inhibitor 3-aminobenzamide during cisplatin nephrotoxicity (Abdelkarim et al. 2001).

\section{Materials and Methods}

\section{Cisplatin nephrotoxicity}

Male C57BL/6 mice aged 8-10 weeks were purchased from Orient Bio (Seongnam, Republic of Korea). All mouse experiments were performed in accordance with the animal protocols approved by the Institutional Animal Care and Use Committee of Jeju National University. Mice were intraperitoneally injected with cisplatin (a single dose of $20 \mathrm{mg} / \mathrm{kg}$ body weight) to induce nephrotoxicity or $0.9 \%$ saline (control). One, two or three days after cisplatin injection, mice were administered PJ34 (R\&D Systems, 2-20 mg/kg body weight per day (Kim et al. 2012)) for inhibition of PARP1 or $0.9 \%$ saline (vehicle) via an intraperitoneal injection. When the mice were anesthetized, the kidneys were collected. The kidneys were either fixed in $4 \%$ paraformaldehyde for histological studies or snap-frozen in liquid nitrogen for biochemical studies. The fixed kidneys were washed with PBS three times for $5 \mathrm{~min}$ each, embedded in paraffin at room temperature, and then cut into $2 \mu \mathrm{m}$ sections using a microtome (catalog no. RM2165; Leica, Bensheim, Germany).

\section{Cell culture}

The kidney proximal tubular cell lines derived from pigs (LLC-PK1) were obtained from American Type Culture Collection (Rockville, MD); and the mouse proximal tubular cell line (MCT) was used as described previously (Haverty et al. 1988, Kim and Padanilam 2013). The MCT and LLC-PK1 cells were maintained in Dulbecco's modified Eagles medium (DMEM)/F-12 medium supplemented with $10 \%$ fetal bovine serum (FBS) and DMEM/high-glucose supplemented with $10 \%$ FBS (Life Technologies, Grand Island, NY) at $37{ }^{\circ} \mathrm{C}$ with $5 \% \mathrm{CO}_{2}$, respectively. The cells were grown until $70 \%$ confluence on culture plates and then changed to serumfree medium. After $18 \mathrm{~h}$ of starvation, the cells were treated with $400 \mu \mathrm{M}$ cisplatin in phosphate-buffered saline (PBS) for $8 \mathrm{~h}$. The cells were also treated with $1 \mu \mathrm{M}$ (high dose) or $100 \mathrm{nM}$ (low dose) PJ34 in PBS (vehicle) at $2 \mathrm{~h}$ after treatment with cisplatin.

\section{ELISA}

PARP activity in kidney tissues was measured by a universal PARP colorimetric assay kit (catalog no. 4677-096-K; Trevigen, Gaithersburg, MD) according to the manufacturer's protocol. Briefly, the kidney tissues were homogenized in 1X PARP buffer on ice. The homogenates were centrifuged at 15,000 rpm for $30 \mathrm{~min}$. The supernatants were added to histone-coated 96 wells and incubated with 1X PARP cocktail for $60 \mathrm{~min}$ at room temperature. After washing wells with $0.1 \%$ Triton $\mathrm{X}-100$ in PBS, PARP activity levels were detected using secondary antibody and substrate, measured in the 
absorbance at $450 \mathrm{~nm}$, and represented as unit of PARP per mg of protein using a typical PARP standard curve. ATP assay was performed on kidney tissues using an ATP fluorometric assay kit (catalog no. K354-100; BioVision, Mountain View, CA) according to the manufacturer's protocol. Briefly, the kidney tissues were homogenized in ATP assay buffer on ice. The homogenates were centrifuged at 15,000 rpm for $30 \mathrm{~min}$. The supernatants were added to 96 wells and incubated with ATP probe, ATP converter and developer in ATP assay buffer for $30 \mathrm{~min}$ at room temperature. After that, ATP levels were measured in the fluorescence $($ excitation/emission $=535 / 587 \mathrm{~nm})$ and represented as nmol of ATP per mg of protein using a typical ATP standard curve. Plasma and culture media were used to measure the level of HMGB1 secretion using a HMGB1 ELISA kit (Catalog no. ST51011; IBL International, Hamburg, Germany) according to the manufacturer's protocol. Briefly, the samples were added to antiHMGB1 antibodies-coated 96 wells and incubated for $24 \mathrm{~h}$ at room temperature. After washing wells with a washing buffer, the 96 wells were incubated with secondary enzyme for $2 \mathrm{~h}$, and then incubate color solution for $30 \mathrm{~min}$. HMGB1 amounts were measured in the absorbance at $450 \mathrm{~nm}$, and represented as unit of HMGB1 per $\mathrm{ml}$ of plasma or culture media using a typical HMGB1 standard curve. All samples were normalized for total protein concentration as assessed by Bradford assay.

\section{Kidney function}

Blood were taken from retro-orbital sinus using heparin-coated capillary tubes at the time indicated in the figures. The tubes were centrifuged at 10,000 rpm for $10 \mathrm{~min}$ using a hematocrit centrifuge. The supernatants were used as plasma. Kidney function was assessed by the concentration of plasma creatinine using QuantiChrom ${ }^{\mathrm{TM}}$ Creatinine Assay kit (Catalog no. DICT500; BioAssay Systems, Hayward, CA) according to the manufacturer's protocol. Briefly, $30 \mu \mathrm{l}$ of plasma were added to 96 wells, and incubated with reagent $\mathrm{A}$ and $\mathrm{B}$ for $5 \mathrm{~min}$ at room temperature. After that, the 96 wells were measured in the absorbance at $510 \mathrm{~nm}$, and represented as $\mathrm{mg}$ of creatinine per dl of plasma or culture media using a typical creatinine standard curve.

\section{Tubular injury}

Hematoxylin and eosin (H\&E)-stained sections were used for tubular injury score as described previously
(Kim et al. 2012). Histological damage of tubular injury was scored by percentage of tubules that displayed tubular necrosis, cast formation and tubular dilation as follows: $0=$ normal, $1=<10 \%, 2=10$ to $25 \%, 3=26$ to $50 \%, 4=51$ to $75 \%$, and $5=>75 \%$. Ten randomly chosen high-power $(\times 200$ magnification $)$ fields per kidney were used for the counting.

\section{Statistical analyses}

Analysis of variance was used to compare data among groups. Differences between vehicle-treated group and PJ34-treated group were assessed by the MannWhitney U-test. $P$ values less than 0.05 were considered statistically significant.

\section{Results}

Postconditioning treatment with PJ34 reduces increased PARP activation during cisplatin nephrotoxicity

To test the effect of a PARP inhibitor in mice undergoing cisplatin nephrotoxicity, we first assessed PARP activity in mouse kidney samples. After cisplatin injection, PARP activity was markedly increased according to time (Fig. 1). However, the kidneys treated with PJ34, a potent PARP inhibitor, from 1 day postinjury showed a significant dose-dependent reduction in the increased PARP activity after cisplatin-induced kidney injury (Fig. 1), indicating that the postconditioning treatment with PJ34 is efficacious in reducing the increased PARP activation during cisplatin nephrotoxicity.

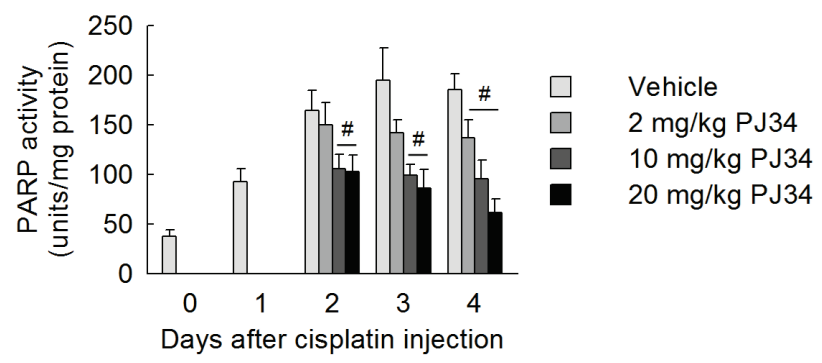

Fig. 1. Postconditioning treatment with PJ34 reduces increased PARP activation during cisplatin nephrotoxicity. Mice were intraperitoneally injected with a single dose of cisplatin to induce nephrotoxicity. One day after cisplatin injection, mice were administered PJ34 (a potent PARP inhibitor) or $0.9 \%$ saline (vehicle) via an intraperitoneal injection. PARP activity was measured by a universal PARP assay kit. Error bars represent SD ( $n=5$ mice in each group). ${ }^{\#} P<0.05$ versus vehicle plus cisplatin 
A

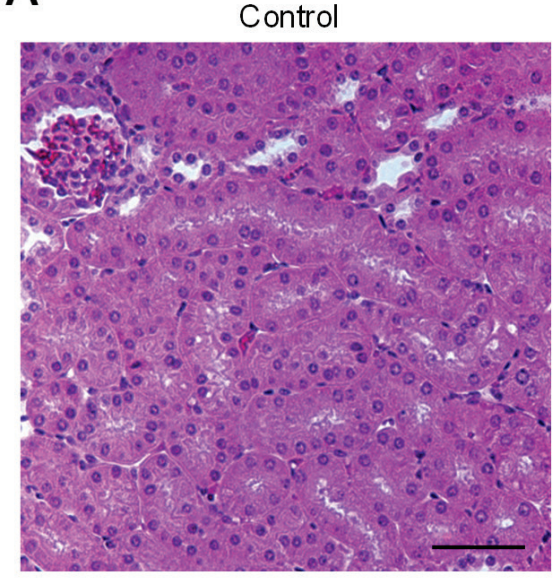

\section{B}

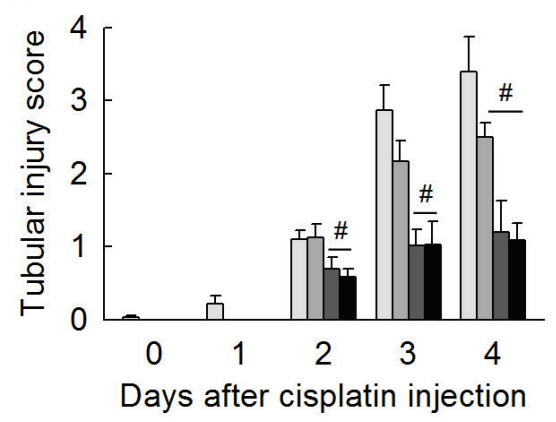

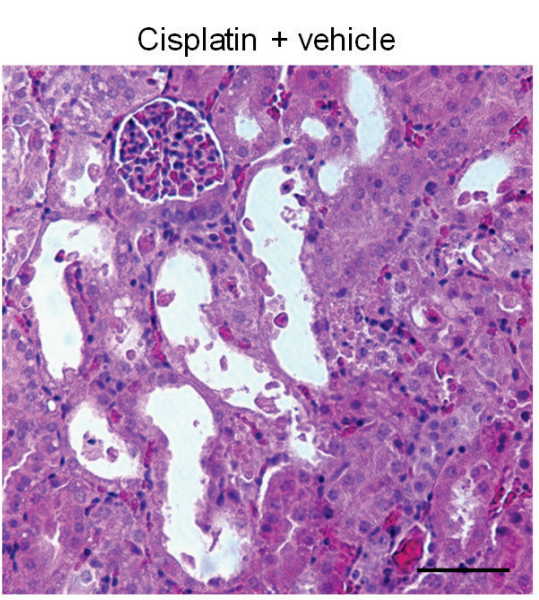

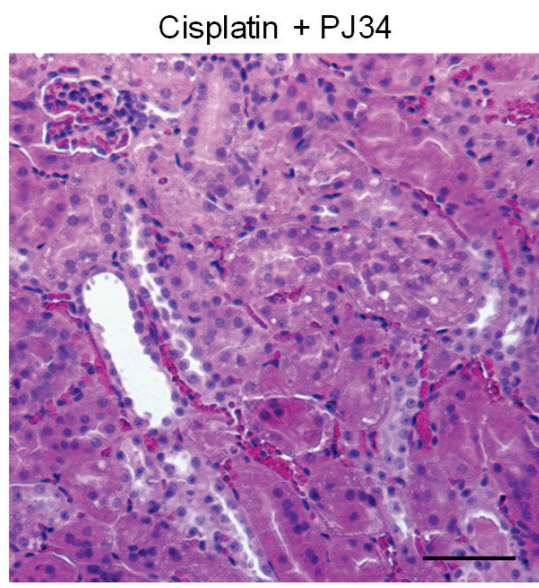

C

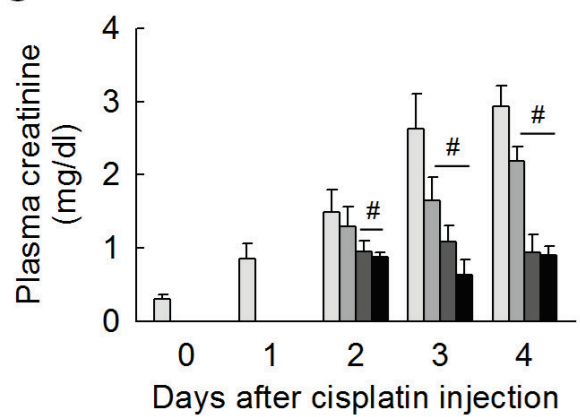

Vehicle

2 mg/kg PJ34

$10 \mathrm{mg} / \mathrm{kg}$ PJ34

$20 \mathrm{mg} / \mathrm{kg}$ PJ34

Fig. 2. Postconditioning treatment with PJ34 attenuates tubular injury and renal dysfunction during cisplatin nephrotoxicity. Mice were intraperitoneally injected with a single dose of cisplatin to induce nephrotoxicity. One day after cisplatin injection, mice were administered PJ34 (a potent PARP inhibitor) or $0.9 \%$ saline (vehicle) via an intraperitoneal injection. (A) Hematoxylin and eosin (H\&E)stained kidney sections in mice treated with $10 \mathrm{mg} / \mathrm{kg}$ body weight of PJ34 or vehicle at 4 days after cisplatin injection. (B) Tubular injury on hematoxylin and eosin (H\&E)-stained kidney sections was scored by counting the percentage of tubules that displayed tubular necrosis, cast formation, and tubular dilation as follows: $0=$ normal; $1=<10 \% ; 2=10$ to $25 \% ; 3=26$ to $50 \% ; 4=51$ to $75 \%$; and $5=>75 \%$. Ten fields ( $\times 200$ magnification) per kidney were used for the counting. (C) Plasma creatinine concentration after cisplatin injection. Error bars represent SD ( $n=5$ mice in each group). ${ }^{\#} P<0.05$ versus vehicle plus cisplatin

Postconditioning treatment with PJ34 diminishes tubular injury and renal dysfunction during cisplatin nephrotoxicity

To determine whether postconditioning treatment with PJ34 prevents the progression of cisplatin nephrotoxicity, we gauged kidney tubular injury during cisplatin nephrotoxicity. The kidneys of cisplatin-injected mice showed a time-dependent increase in tubular injury score at 1, 2, 3, and 4 days after the onset of injury, compared to that at 0 day; whereas treatment with PJ34 from 1 day post-injury dose-dependently attenuated the increase in tubular injury score at 2, 3, and 4 days after cisplatin injection (Fig. 2, A and B). To determine whether postconditioning treatment with $\mathrm{PJ} 34$ reduces renal dysfunction induced by cisplatin nephrotoxicity, we measured the concentration of plasma creatinine. Consistent with the result of tubular injury score, postconditioning treatment with PJ34 dose-dependently suppressed the increment of plasma creatinine concentration at 2, 3, and 4 days after cisplatin injection (Fig. 2C). These data suggest that postconditioning treatment with PJ34 prevents the progression of cisplatin nephrotoxicity. In addition, we tested the effect of treatment with PJ34 according to time post-injury. Cisplatin-induced tubular injury and renal dysfunction were more significantly attenuated by earlier treatment with PJ34 after cisplatin injection (Fig. 3, A and B).

Postconditioning treatment with PJ34 attenuates ATP depletion during cisplatin nephrotoxicity

Since excessive activation of PARP depletes energy stores such as ATP (Ha and Snyder 1999), we measured the concentration of ATP in mouse kidneys during cisplatin nephrotoxicity. Cisplatin-injected mouse kidneys showed a decrease in ATP concentrations in a time-dependent manner (Fig. 4). However, 
postconditioning treatment with PJ34 significantly inhibited the decrease in ATP concentrations in a dosedependent manner during cisplatin nephrotoxicity
(Fig. 4). These data suggest that the postconditioning treatment with PJ34 efficaciously attenuates ATP depletion during cisplatin nephrotoxicity.
A

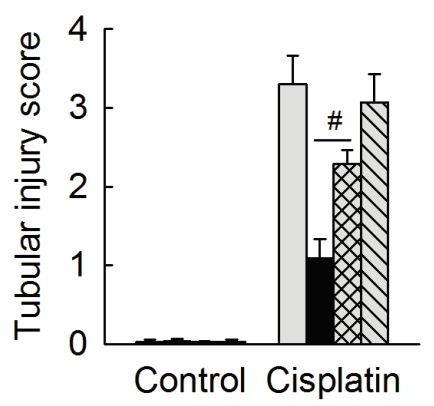

Four days after cisplatin injection
B

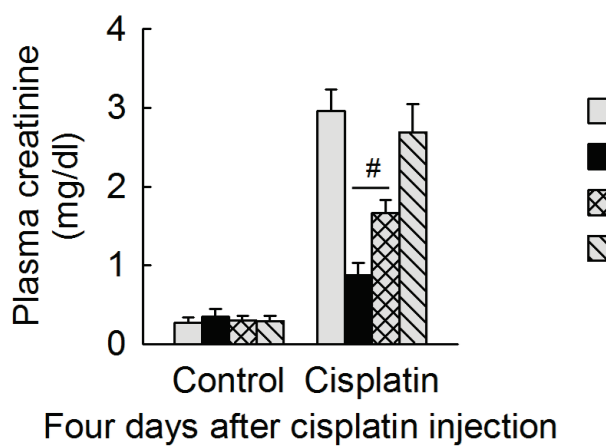

Vehicle

PJ34 at 1 day post-injury

PJ34 at 2 days post-injury

PJ34 at 3 days post-injury

Fig. 3. Treatment with PJ34 post injury diminishes cisplatin nephrotoxicity in a time-dependent manner. Mice were intraperitoneally injected with a single dose of cisplatin to induce nephrotoxicity or $0.9 \%$ saline (control). One, two or three days after cisplatin injection, mice were administered PJ34 (a potent PARP inhibitor) or $0.9 \%$ saline (vehicle) via an intraperitoneal injection. (A) Tubular injury on H\&E-stained kidney sections was scored by counting the percentage of tubules that displayed tubular necrosis, cast formation, and tubular dilation. (B) Plasma creatinine concentration after cisplatin injection. Error bars represent SD ( $n=5$ mice in each group). ${ }^{\#} P<0.05$ versus vehicle plus cisplatin

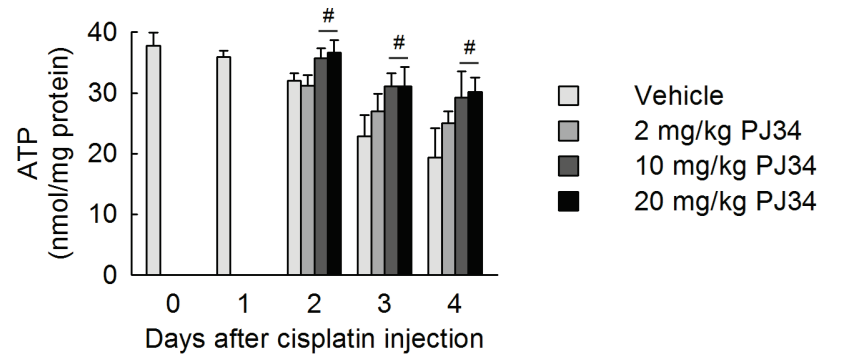

Fig. 4. Postconditioning treatment with PJ34 reduces ATP depletion during cisplatin nephrotoxicity. Mice were intraperitoneally injected with a single dose of cisplatin to induce nephrotoxicity. One day after cisplatin injection, mice were administered PJ34 (a potent PARP inhibitor) or $0.9 \%$ saline (vehicle) via an intraperitoneal injection. The level of ATP was measured by an ATP assay kit. Error bars represent SD ( $n=5$ mice in each group). ${ }^{*} P<0.05$ versus vehicle plus cisplatin

Postconditioning treatment with PJ34 suppresses HMGB1 release from kidney proximal tubular cells during cisplatin nephrotoxicity

PARP activation can mediate necrotic cell death by ATP depletion (Ha and Snyder 1999), and necrotic cells passively release HMGB1 chromatin protein (RovereQuerini et al. 2004). Herein we detected an increase of systemic HMGB1 levels in mouse plasma during cisplatin nephrotoxicity (Fig. 5). However, postconditioning treatment with PJ34 significantly reduced the increase in plasma HMGB1 levels at 2, 3, and 4 days after cisplatin injury (Fig. 5), indicating that PARP activation leads to

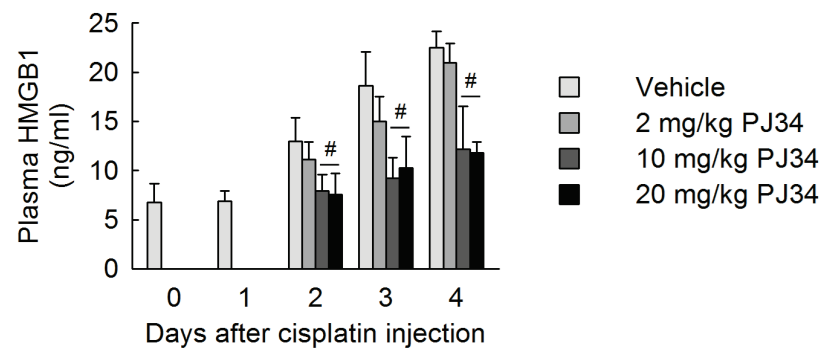

Fig. 5. Postconditioning treatment with PJ34 attenuates systemic HMGB1 release during cisplatin nephrotoxicity. Mice were intraperitoneally injected with a single dose of cisplatin to induce nephrotoxicity. One day after cisplatin injection, mice were administered PJ34 (a potent PARP inhibitor) or $0.9 \%$ saline (vehicle) via an intraperitoneal injection. Plasma was used to measure the level of HMGB1 secretion using a HMGB1 ELISA kit. Error bars represent SD ( $n=5$ mice in each group). ${ }^{\#} P<0.05$ versus vehicle plus cisplatin

systemic HMGB1 release during cisplatin nephrotoxicity. To confirm our in vivo finding that PARP activation is involved in systemic HMGB1 release, we used kidney proximal tubule epithelial cell lines. After $8 \mathrm{~h}$ of cisplatin injury, the level of endogenous HMGB1 was dramatically increased in LLC-PK1 and MCT cell culture media. However, postconditioning treatment with PJ34 significantly attenuated the increase in extracellular HMGB1 level after $8 \mathrm{~h}$ of cisplatin injury (Fig. 6, A and B). These data suggest that PARP1 activation is required for HMGB1 release from kidney proximal tubule epithelial cells during cisplatin nephrotoxicity. 

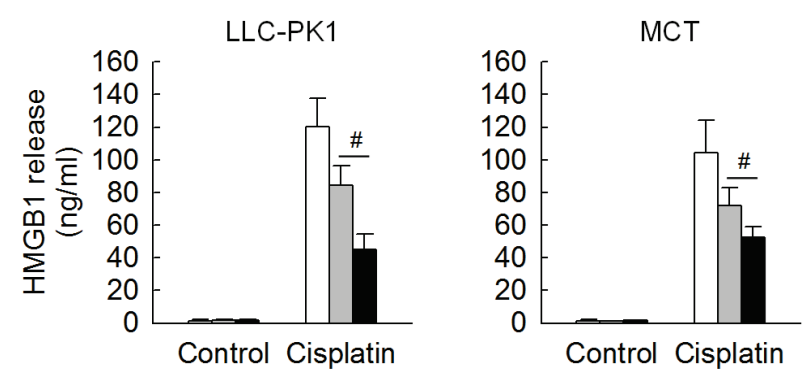

\section{Discussion}

Cisplatin nephrotoxicity is implicated in acute kidney injury characterized by renal dysfunction and tubular cell death. Despite being the focus of active investigations so far, there is an open question in our understanding of the exact pathogenesis of cisplatin nephrotoxicity. Recent studies have emphasized on inflammation and apoptotic cell death, considered to be the major contributors to kidney injury. The present data demonstrated a novel finding that 1) cisplatin induces necrosis and HMGB1 release in an in vivo model of cisplatin nephrotoxicity, 2) HMGB1 released by cisplatin is involved in PARP activation, and further 3) PARP inhibition after treatment with cisplatin ameliorates nephrotoxicity in mice. These data suggest that necrosis is a major determinant of cisplatin nephrotoxicity, supporting the finding that the predominant lesion is acute necrosis in patients with cisplatin acute kidney injury (Yao et al. 2007).

Kidney tissue damage, characterized by necrotic and apoptotic cell death, is a common histopathological feature of cisplatin nephrotoxicity (Pabla and Dong 2008). Especially, kidney proximal tubular cells are the major sites of cell death induced by cisplatin (Shino et al. 2003). Earlier observations in cultured kidney tubular cells by Lieberthal et al. (1996) suggested that apoptosis is observed at low concentrations of cisplatin for a long time, whereas necrosis occurs upon high concentrations of cisplatin for a short time. In in vivo animal models, both apoptosis and necrosis are induced in kidney tubules after cisplatin administration (Kim et al. 2012). Nevertheless, cisplatin-induced apoptotic cell death has been investigated further. The previous study demonstrated that PARP gene deletion prevents tubular necrosis following cisplatin administration in mice (Kim et al. 2012). Consistent with the previous study, the present study also showed that PARP inhibition by
Fig. 6. Postconditioning treatment with PJ34 diminishes HMGB1 release from kidney proximal tubular cells. After $18 \mathrm{~h}$ of starvation, LLC-PK1 and MCT cells were treated with $400 \mu \mathrm{M}$ cisplatin for $\begin{array}{ll}\text { Vehicle } & 8 \mathrm{~h} . \text { The cells were also treated with a } \\ \text { Low dose of PJ34 } & \text { high or low dose of PJ34 at } 2 \mathrm{~h} \text { after }\end{array}$ High dose of PJ34 treatment with cisplatin. After $8 \mathrm{~h}$ of High dose of PJ34 cisplatin injury, cell culture media were used to measure the level of HMGB1 secretion using a HMGB1 ELISA kit. Error bars represent SD $(n=3$ experiments). ${ }^{\#} P<0.05$ versus vehicle plus cisplatin postconditioning reduced kidney tubular necrosis, represented by tubular injury score and ATP depletion, during cisplatin nephrotoxicity. These data suggest that necrosis is a major determinant of cisplatin nephrotoxicity, supported by the finding that the predominant lesion is acute necrosis in patients with cisplatin acute kidney injury (Yao et al. 2007).

Damaged tubules can release alarming factors such as HMGB1 to activate inflammatory response (Goligorsky 2011). HMGB1 is a nuclear factor that is involved in transcriptional activation and DNA folding (Javaherian et al. 1978), and when it is released into the extracellular space, it can be a cytokine or ligand that triggers toll-like receptor (TLR) signaling, which is a critical mediator of innate immune responses to injury and infection (Yang et al. 2005). Consistent with the previous other study (Zhang et al. 2008), our data have demonstrated that cisplatin increased TLR4 expression in kidneys in our mouse model; but not shown no significant difference in TLR4 expression between vehicle- and PJ34-treated kidneys during cisplatin nephrotoxicity (data not shown). Although endogenous HMGB1 does not play a significant role in cellular responses to cisplatin (Wei et al. 2003), extracellular HMGB1 released from necrotic cells in kidney tubules may contribute to inflammatory responses during cisplatin nephrotoxicity. Cisplatin-induced inflammatory responses contribute to the development of kidney tissue damage and kidney dysfunction, which causes acute kidney injury (Zhang et al. 2008). In kidney ischemiareperfusion injury, extracellular HMGB1 mediates kidney tubular damage via the toll-like receptor 4 pathway (Wu et al. 2010). In mouse embryonic fibroblasts, PARP gene deletion reduces HMGB1 secretion (Ditsworth et al. 2007). The present data show for the first time that PARP inhibition results in reduced release of HMGB1 from kidney proximal tubular cells during cisplatin nephrotoxicity, suggesting that PARP-dependent 
HMGB1 release from kidney proximal tubular cells may contribute to inflammation during cisplatin nephrotoxicity.

In conclusion, the results of this study show that PARP overactivation in cisplatin-injected kidneys leads to cisplatin nephrotoxicity, and further, overactivated PARP contributes to HMGB1 release from proximal tubular cells through induction of necrotic cell death during cisplatin nephrotoxicity. PARP may be a pivotal molecule in cisplatin-mediated promotion of necrotic effects.

\section{Conflict of Interest}

There is no conflict of interest.

\section{Acknowledgements}

This work was supported by the Academic Research Foundation of Jeju National University Institute of Medical Science in 2014.

\section{References}

ABDELKARIM GE, GERTZ K, HARMS C, KATCHANOV J, DIRNAGL U, SZABO C, ENDRES M: Protective effects of PJ34, a novel, potent inhibitor of poly(ADP-ribose) polymerase (PARP) in in vitro and in vivo models of stroke. Int J Mol Med 7: 255-260, 2001.

ARANY I, SAFIRSTEIN RL: Cisplatin nephrotoxicity. Semin Nephrol 23: 460-464, 2003.

BEYER J, RICK O, WEINKNECHT S, KINGREEN D, LENZ K, SIEGERT W: Nephrotoxicity after high-dose carboplatin, etoposide and ifosfamide in germ-cell tumors: incidence and implications for hematologic recovery and clinical outcome. Bone Marrow Transplant 20: 813-819, 1997.

DEVALARAJA-NARASHIMHA K, PADANILAM BJ: PARP-1 inhibits glycolysis in ischemic kidneys. J Am Soc Nephrol 20: 95-103, 2009.

DITSWORTH D, ZONG WX, THOMPSON CB: Activation of poly(ADP)-ribose polymerase (PARP-1) induces release of the pro-inflammatory mediator HMGB1 from the nucleus. J Biol Chem 282: 17845-17854, 2007.

GOLIGORSKY MS: TLR4 and HMGB1: partners in crime? Kidney Int 80: 450-452, 2011.

HA HC, SNYDER SH: Poly(ADP-ribose) polymerase is a mediator of necrotic cell death by ATP depletion. Proc Natl Acad Sci U S A 96: 13978-13982, 1999.

HAVERTY TP, KELLY CJ, HINES WH, AMENTA PS, WATANABE M, HARPER RA, KEFALIDES NA, NEILSON EG: Characterization of a renal tubular epithelial cell line which secretes the autologous target antigen of autoimmune experimental interstitial nephritis. $J$ Cell Biol 107: 1359-1368, 1988.

JAVAHERIAN K, LIU JF, WANG JC: Nonhistone proteins HMG1 and HMG2 change the DNA helical structure. Science 199: 1345-1346, 1978.

KIM J, PADANILAM BJ: Loss of poly(ADP-ribose) polymerase 1 attenuates renal fibrosis and inflammation during unilateral ureteral obstruction. Am J Physiol 301: F450-F459, 2011.

KIM J, PADANILAM BJ: Renal nerves drive interstitial fibrogenesis in obstructive nephropathy. J Am Soc Nephrol 24: 229-242, 2013.

KIM J, LONG KE, TANG K, PADANILAM BJ: Poly(ADP-ribose) polymerase 1 activation is required for cisplatin nephrotoxicity. Kidney Int 82: 193-203, 2012.

KRAUS WL, LIS JT: PARP goes transcription. Cell 113: 677-683, 2003.

KRISHNAKUMAR R, GAMBLE MJ, FRIZZELL KM, BERROCAL JG, KININIS M, KRAUS WL: Reciprocal binding of PARP-1 and histone $\mathrm{H} 1$ at promoters specifies transcriptional outcomes. Science 319: 819-821, 2008.

LIEBERTHAL W, TRIACA V, LEVINE J: Mechanisms of death induced by cisplatin in proximal tubular epithelial cells: apoptosis vs. necrosis. Am J Physiol 270: F700-F708, 1996.

LOTZE MT, TRACEY KJ: High-mobility group box 1 protein (HMGB1): nuclear weapon in the immune arsenal. Nat Rev Immunol 5: 331-342, 2005.

MARTIN DR, LEWINGTON AJ, HAMMERMAN MR, PADANILAM BJ: Inhibition of poly(ADP-ribose) polymerase attenuates ischemic renal injury in rats. Am J Physiol Regul Integr Comp Physiol 279: R1834R1840, 2000. 
MOUBARAK RS, YUSTE VJ, ARTUS C, BOUHARROUR A, GREER PA, MENISSIER-DE MURCIA J, SUSIN SA: Sequential activation of poly(ADP-ribose) polymerase 1, calpains, and Bax is essential in apoptosis-inducing factor-mediated programmed necrosis. Mol Cell Biol 27: 4844-4862, 2007.

PABLA N, DONG Z: Cisplatin nephrotoxicity: mechanisms and renoprotective strategies. Kidney Int 73: 994-1007, 2008.

ROVERE-QUERINI P, CAPOBIANCO A, SCAFFIDI P, VALENTINIS B, CATALANOTTI F, GIAZZON M, DUMITRIU IE, MULLER S, IANNACONE M, TRAVERSARI C, BIANCHI ME, MANFREDI AA: HMGB1 is an endogenous immune adjuvant released by necrotic cells. EMBO Rep 5: 825-830, 2004.

SCAFFIDI P, MISTELI T, BIANCHI ME: Release of chromatin protein HMGB1 by necrotic cells triggers inflammation. Nature 418: 191-195, 2002.

SHEVALYE H, STAVNIICHUK R, XU W, ZHANG J, LUPACHYK S, MAKSIMCHYK Y, DREL VR, FLOYD EZ, SLUSHER B, OBROSOVA IG: Poly(ADP-ribose) polymerase (PARP) inhibition counteracts multiple manifestations of kidney disease in long-term streptozotocin-diabetic rat model. Biochem Pharmacol 79: 1007$1014,2010$.

SHINO Y, ITOH Y, KUBOTA T, YANO T, SENDO T, OISHI R: Role of poly(ADP-ribose)polymerase in cisplatininduced injury in LLC-PK1 cells. Free Radic Biol Med 35: 966-977, 2003.

SIDDIK ZH: Cisplatin: mode of cytotoxic action and molecular basis of resistance. Oncogene 22: 7265-7279, 2003.

STARK JJ, HOWEL SB: Nephrotoxicity of cis-platinum (II) dichlorodiammine. Clin Pharmacol Ther 23: 461-466, 1978.

THOMAS JO, TRAVERS AA: HMG1 and 2, and related 'architectural' DNA-binding proteins. Trends Biochem Sci 26: 167-174, 2001.

WANG D, LIPPARD SJ: Cellular processing of platinum anticancer drugs. Nat Rev Drug Discov 4: 307-320, 2005.

WEI M, BURENKOVA O, LIPPARD SJ: Cisplatin sensitivity in Hmbg1-/- and Hmbg1+/+ mouse cells. J Biol Chem 278: 1769-1773, 2003.

WU H, MA J, WANG P, CORPUZ TM, PANCHAPAKESAN U, WYBURN KR, CHADBAN SJ: HMGB1 contributes to kidney ischemia reperfusion injury. J Am Soc Nephrol 21: 1878-1890, 2010.

YANG H, WANG H, CZURA CJ, TRACEY KJ: The cytokine activity of HMGB1. J Leukoc Biol 78: 1-8, 2005.

YAO X, PANICHPISAL K, KURTZMAN N, NUGENT K: Cisplatin nephrotoxicity: a review. Am J Med Sci 334: 115 124, 2007.

ZHANG B, RAMESH G, UEMATSU S, AKIRA S, REEVES WB: TLR4 signaling mediates inflammation and tissue injury in nephrotoxicity. J Am Soc Nephrol 19: 923-932, 2008. 